\title{
Leistungsnachweis statt Leistungsgutschein
}

\section{Jürg Unger-Köppel}

Dr. med., Mitglied des FMH-Zentralvorstandes, Departementsverantwortlicher Stationäre Versorgung und Tarife

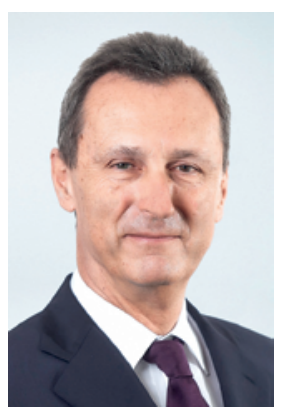

Ein älterer Referent aus Deutschland sagte am DRG Forum: «Früher gab es einen ungeschriebenen Gesellschaftsvertrag: Die Ärzte schauen uns und wir schauen für die Ärzte. Aber dieser Vertrag gegenüber uns Ärzten gilt heute nicht mehr.» Die Nachwirkungen dieser Vertragskündigung durch die Gesellschaft sind gut spürbar: Sitzt man am Tisch mit jenen, die die Leistungen bezahlen, herrscht vielfach Misstrauen gegenüber unserem ärztlichen Tun, und als Folge muss die erbrachte Leistung immer detaillierter nachgewiesen werden. Das Resultat sind über 1000 DRGs, ungezählte CHOPs und ungefähr 2500 Positionen im TARDOC. Gleichzeitig frisst die damit erforderliche Leistungserfassung unsere Zeit am Patienten weg. Abends erfassen wir die Leistungsminuten des Tages patientenscharf in unseren Abrechnungssystemen, damit die nächste Version der DRG noch besser wird, statt dass wir eine Vertrauen schaffende «Bettmümpfeli-Visite» am Krankenbett machen können. Kommen wir dann spätabends müde nach Hause, kontrollieren wir selbst sehr genau die Rechnung des letzten Autoservice auf deren Plausibilität.

\section{Auch im zukünftigen Rehabilitationstarif STReha sollen Tagespauschalen zugunsten eines Leistungsnachweises abgeschafft werden.}

Seit 2018 gelten auch in der Psychiatrie die Tagespauschalen nicht mehr, die den Erfassungsaufwand minimal hielten (Patient da: ja/nein). Nur in der Rehabilitation genügt heute noch der Nachweis, dass der Patient da war, um die Tagespauschale zu bekommen. Zuvor muss natürlich aufwendig die Indikation zur stationären Rehabilitation aufgrund des Zustandes des Patienten und seines Rehabilitationspotentials für die Kassen überzeugend nachgewiesen werden. Aber was mit dem Patienten Tag für Tag gemacht wird, muss heute in der Rehabilitation noch nicht gezeigt werden, um von den Krankenkassen bezahlt zu werden. Damit wird gleichsam der Platz auf der Spitalliste zum Leistungsgutschein, ohne dass man den genauen Gegenwert kennt.

\section{Gemeinsam mit H+ konnte die FMH erreichen,} dass der Erfassungsaufwand nicht überbordet.

Gemäss dem aktuellen politischen Trend «Misstraue der Ärzteschaft» soll im zukünftigen Rehabilitationstarif STReha natürlich ebenfalls ein Leistungsnachweis erbracht werden. Damit beginnt auch in der Rehabilitation die Diskussion um die CHOPS und die genaue Kostenabbildung. Schnell spalten sich jene, die ein möglichst einfaches System wünschen, von jenen, die eine möglichst genaue Erfassung fordern - zwei grundlegend gegensätzliche Ziele, die in allen Tarifsystemen zu beobachten sind.

Gemeinsam mit den betroffenen Fachgesellschaften hat die Abteilung stationäre Versorgung und Tarife der FMH in über zweijähriger Arbeit die Basis- und Zusatzleistungen in der Rehabilitation definiert und den Partnern der SwissDRG AG und dem BFS zwecks Etablierung in der CHOP unterbreitet. Schlussendlich konnte die FMH ihre Partner erfolgreich von einem Mittelweg als Kompromiss überzeugen. Dieser Weg erfordert einen gewissen Erfassungsaufwand, versucht aber, diesen in Grenzen zu halten (vgl. dazu z.B. Bruno Trezzini und Beatrix Meyer in der SÄZ 2019;100(6): 161-2). Ende April 2019 wurde diese gemeinsame Haltung der Verhandlungspartner anlässlich einer Sitzung im Bundesamt für Statistik BFS erneut bestätigt. Von den Kassen und Kantonen wurde sehr klar eine Transparenz bezüglich der erbrachten Leistung eingefordert. Die FMH konnte aber, unterstützt durch $\mathrm{H}+$, erreichen, dass der Erfassungsaufwand nicht überbordet. 